\title{
SABRE: A Time Efficient Semi-automated Regional Parcellation Method for Structural Magnetic Resonance Brain Images
}

\author{
L.A Dade ${ }^{1,2}$, F.Q. Gao ${ }^{2}$, N. Kovacevic ${ }^{2}$, P. Roy $^{2}$, C. Rockel $^{2}$, C.M. O'Toole ${ }^{1,2}$,
} A. Quddus $^{2}$, A. Feinstein ${ }^{2}$, B. Levine ${ }^{1,2}$, and S.E. Black ${ }^{1,2}$

${ }^{1}$ Rotman Research Institute, Baycrest Centre for Geriatric Care,

3160 Bathurst Street Toronto, Ontario, Canada M4S 1V5

${ }^{2}$ Sunnybrook and Women's College Health Science Centre

2075 Bayview Avenue, Toronto, ON M4N 3M5

University of Toronto, Toronto Ontario, Canada

\begin{abstract}
Structural MR brain imaging is important in the understanding of regional brain changes in healthy aging and in disease processes. Current methods are often labour-intensive, requiring hours of manual tracing. SABRE (Semi-automatic Brain Region Extraction) is a new technique that allows rapid and reliable parcellation of cortical and subcortical brain regions. Utilization of SABRE in conjunction with a validated tissue-compartment segmentation program [1] can provide measures of gray matter, white matter, ventricular $\mathrm{CSF}$, and sulcal CSF measures for designated brain regions. We present data showing high inter-rater reliably for all regions, with correlations between 0.95 - 0.99. Regional tissue measures of healthy young and healthy older adults were also examined, demonstrating the sensitivity of this protocol to brain volume changes that occur with aging.
\end{abstract}

\section{Introduction}

We present a semi-automated brain region extraction program (SABRE) that, when applied in conjunction with a tissue segmentation program[1], can provide rapid gray, white, ventricular and sulcal CSF measures for 26 specified brain regions. Two studies were completed: one to evaluate the inter-rater reliability of the method, and a second to examine construct validity of SABRE by determining if it could detect known tissue atrophy effects that occur with normal aging.

\section{Methods}

These two studies were approved by the ethics review board. All subjects gave informed consent, and none had a history of neurological disease. MR images were acquired on a $1.5 \mathrm{~T}$ Signa GE scanner. Each subject had a T1-weighted, protondensity (PD) and T2-weighted scan.

SABRE involves four steps: 1) Transformation of the T1 scan to ac-pc aligned space, 2) demarcation of 13 landmarks (20 minutes), 3) automatic creation of an individual Talairach grid, and 4) running of the automated SABRE program to create 
a mask delineating 24 (12 left, 12 right) brain regions (e.g. lateral and medial inferior frontal lobe, anterior temporal lobe, superior parietal lobe). An automated medial temporal lobe mask is also created based on Talairach grid divisions adding left and right medial temporal measures. This 26 brain region mask is then applied to the tissue segmented T1 image previously created using the Kovacevic method[1].

For the inter-rater reliability study, scans of 10 young adults (age: $X=28.4$, sd= 5.7) and 11 older adults $(X=78.3, \mathrm{sd}=10.8)$ were used. Two trained and experienced operators (FQG and PR) independently applied the SABRE method to the scans. Intraclass correlation estimates (ICCs) for single ratings, with raters as a fixed factor, was used to examine inter-rater reliability across brain regions.

To examine the sensitivity of SABRE to age related volume effects, scans from 10 younger $(X=28.4$, sd $=5.8)$ and 10 older adults $(X=66.8, s d=8.7)$ were used. Repeated measures ANOVA was applied and paired t-tests were used as post hocs to evaluate regional tissue volume differences between young and old groups.

\section{Results}

No significant differences were found between left and right regions; therefore regions were collapsed across hemispheres. For inter-rater reliability, ICCs for total regional volumes $(0.95-0$.99), and for specific tissue compartment volumes (gray, white, sulcal and ventricular CSF) were all high (0.97-0.99).

Repeated measures analysis of group (young vs old) by region, revealed a significant interaction $\left(\mathrm{F}_{1,44}=10.0, \mathrm{p}<.001\right)$. Post hoc analyses indicated significant atrophy in the older group in the majority of areas including frontal, temporal, and parietal gray matter. Non-significant differences occurred only in lateral and medial inferior frontal white matter, lateral superior white matter, occipital gray matter, and all tissues of the basal ganglia and thalamus region.

\section{Conclusion}

Relative to other methods SABRE is a rapid and reliable technique for obtaining in vivo regional brain tissue volumes. The studies presented show that it has high interrater reliability and is sensitive to the volume changes that occur with normal aging.

\section{References}

1. Kovacevic, N., Lobaugh, N.J., Bronskill, M.J., Levine, B., Feinstein, A. \& Black, S.E.: A Robust Method for extraction and automatic segmentation of brain images. Neuroimage. 17 (2002) 1087-1100 